\title{
Evaluación de la amenaza natural en ambiente semiárido, sustentada en la geomorfología y el modelamiento de índices topográficos. Sala- manca, Región de Coquimbo, Chile*.
}

\author{
María Victoria Soto Bäuerle \\ Departamento de Geografía, Facultad de Arquitectura y Urbanismo, Universidad de Chile, \\ Santiago. Chile. \\ mvsoto@uchilefau.cl

\section{Michael Märker} \\ Dipartimento di Scienze delle Produzioni Vegetali, del Suolo e dell'Ambiente Agroforesta- \\ le (DIPSA), Universitá degli studi di Firenze, Florencia, Italia. \\ Geographisches Institut der Eberhard Karls Universität Tübingen, Tübingen, Alemania.
}

michael.maerker@unifi.it

\section{Joselyn Arriagada González}

Departamento de Geografía, Facultad de Arquitectura y Urbanismo, Universidad de Chile, Santiago, Chile.

joarriag@uchile.cl

\section{Carmen Paz Castro Correa}

Departamento de Geografía, Facultad de Arquitectura y Urbanismo, Universidad de Chile, Santiago, Chile.

cpcastro@uchile.cl

Giuliano Rodolfi

Universitá degli studi di Firenze, Florencia, Italia.

giuliano.rodolfi@unifi.it

\begin{abstract}
RESUMEN
En el marco de las trasformaciones territoriales en el valle del río Choapa, se analizan los procesos dinámicos de la cuenca, y cómo éstos pueden verse modificados debido a las intervenciones antrópicas. En el dominio semiárido, tanto el paisaje natural como el antropizado, presentan una respuesta dinámica actual de diversa intensidad e impacto ante eventos pluviométricos, principalmente al fenómeno de El Niño. La agricultura intensiva que se está desarrollando en laderas, conos, glacis, es una actividad incipiente pero en incremento, generando transformaciones topográficas, de cobertura vegetal y de suelo. Se analiza la geomorfología como formas heredadas y sus procesos dinámicos. Junto con la carta geomorfológica, se aplican modelos topográficos que complementan el análisis morfológico y contribuyen a deducir una condición dinámica. A partir de ello se modela la amenaza natural, para dos sectores específicos, Salamanca y Panguesillo. Si bien el marco geomorfológico es similar, no lo es en cuanto a uso del suelo y nivel de amenaza natural.
\end{abstract}

Palabras Clave: sistemas de vertientes; conos; glacis, amenaza

* $\quad$ Proyecto FONDECYT No 1071098 
Natural hazard assessment in semi-arid environment, based on the geomorphology and modeling of topographic index. Salamanca, Coquimbo Region, Chile.

\begin{abstract}
In the framework of territorial transformations in the Choapa river valley, we analyze the dynamics of geomorphic processes on basin scale. Special attention is given to changes due to human activities. In semiarid environments, both natural and anthropic landscapes have an immediate dynamic response to varying intensity and impact of rainfall events such as the phenomenon of El Niño. Intensive farming on hillsides, cones and glacis, is an emerging industry, inducing transformations of topography, vegetation and soil. We analyze the current geomorphology characterized by inherited forms, features and their process dynamics. Geomorphological and topographical models are applied to assess the relevant morphodynamics. The models yield information on the natural hazard of mass movements and fluvially triggred erosion processes for the two study areas Salamanca and Panguesillo.
\end{abstract}

Key words: slope system; aluvial fan; glacis; hazard

\section{INTRODUCCIÓN}

El ambiente semiárido que caracteriza al valle del río Choapa, ha significado que existan actividades agrícolas desde tiempos históricos y el asentamiento y consolidación de la cuidad de Salamanca, como de diversos poblados menores junto a las riberas del río; este poblamiento lineal se proyecta hasta la localidad de Batuco, ésta última, inserta ya en el dominio periglacial de altura.

El paisaje analizado corresponde a lo que ARAYA-VERGARA (1980) estableció como subtropical seco, semiárido, muy marcado por rasgos heredados y relictos. El valle presenta un sistema de laderas y de formas depositacionales asociadas, que dan cuenta de la acción del agua durante tiempos geológicos diferentes del actual, y que se constituyen, desde la perspectiva de la geodinámica y transferencia de masa actual, en los emisores, con un funcionamiento esporádico, asociado a los eventos El Niño. DEANE \& WAYLEN (2009) estudiaron estos eventos en las dos principales ciudades de la cuenca, Illapel y Salamaca. A partir de datos de precipitaciones diarias entre los años 1974 y 1998, establecieron un umbral de precipitaciones $(25 \mathrm{~mm})$ que si bien fue realizado con fines biogeográficos específicos, da cuenta que en eventos El Niño ocurren precipitaciones concentradas a partir del umbral, hasta 3 veces durante los meses de mayo y septiembre. A partir de tales datos se deriva la ocurrencia de eventos muy concentrados y de alta intensidad, que funcionarían como agentes geodinámicos, sobre todo en el dominio semiárido donde predominan formas relictas, como glacis (pediments), conos y terrazas. En este contexto ANDREW et al., (2008) demostraron cómo eventos climáticos intensos y concentrados generan una importante condición de aporte de sedimentos, los que son conducidos por los talwegs y regueros de erosión de las superficies de glacis hacia las terrazas y a la red fluvial, pudiendo transformar en el período de 20 años el canal principal meandrante, en anastomosado. La condición dinámica actual de los ambientes áridos y semiáridos, como formas heredadas fue analizada por STRUDLEY et al., (2006) comprobando la estrecha relación existente entre la meteorización del substrato y la presencia de tors en las superficies de glacis y piedmonts, los que estarían estrechamente vinculados con el transporte 
de sedimentos por condiciones climáticas y tectónicas. ARAYA-VERGARA (2000) ha descrito este tipo de fenómenos en laderas graníticas de Chile central, identificando y caracterizando superficies modeladas por procesos de erosión intensa, generando la exhumación de rocas y la alteración de las mismas.

En el escenario de cambios de paisaje por la acción antrópica y los de cambio climático, la modelación realizada por GARREAUD et al., (2008) da cuenta de condiciones apropiadas para la generación de procesos geodinámicos de mayor intensidad, recurrencia espacial y frecuencia temporal, que en la actualidad. Esta situación coindice con lo observado en el valle del Copiapó por CASTRO et al., (2009), donde además se concluye la existencia de condiciones de amenaza natural asociada a la pluviosidad y el impacto del uso del suelo en cultivos de parronales.

Además, se debe de considerar los cambios del paisaje urbano, que si bien en el valle ha sido poco expansivo, las actividades agrícolas y mineras en desarrollo y crecimiento, indican una tendencia al aumento de la población y de los centros poblados, principalmente de Salamanca.

A partir de las condiciones dinámicas del sistema natural y del uso del mismo, es posible introducir el concepto del riesgo. Este concepto, ampliamente mal usado como sinónimo de peligro, debe ser entendido como la exposición a procesos naturales de una comunidad (HELMHOLTZ ASSOCIATION 2007). Desde el punto de vista de la planificación territorial, el riesgo deriva de la exposición en áreas propensas. Actualmente, en el contexto del paradigma del enfoque holístico, riesgo y desastre son considerados como un problema del desarrollo aún no resuelto, es decir un proceso socio ambiental (CARDONA 2009). La vulnerabilidad, que es la propensión a los riesgos, es una condición derivada de la interacción de un conjunto de factores que interactúan entre si, conformando un sistema complejo (la falta de planificación con enfoque de cuenca hidrográfica, la intensificación del uso de la tierra, etc.). En el enfoque holístico, consecuentemente la base del sistema está constituido por la amenaza, que es el fenómeno natural que puede afectar a la sociedad.

Ello implica entonces, aumento de la vulnerabilidad y del riesgo asociado a la amenaza natural vinculada al emplazamiento de las entidades pobladas y al aumento de la misma por la acción detonadora y sinérgica de las acciones antrópicas. El caso más representativo corresponde a transformaciones topográficas del terreno, principalmente a través de movimientos de tierra a fin de crear terrazas para producción agrícola; estos procesos han modificado los perfiles de equilibrio de las formas naturales. Cuando ocurren los eventos El Niño, existen condiciones favorables con masa detrítica disponible para ser incorporada en los cursos de agua, aumentando así la torrencialidad de los mismos (SOTO et al., 2009). Actualmente, la incorporación de suelos en cotas más elevadas, correspondiente a terrazas residuales está en desarrollo, con la consecuente modificación topográfica del terreno, y los impactos ya descritos, pero de mayor intensidad, por la pendiente y la conformación sedimentológica de tales formas.

Consecuentemente, en el área de estudio existen condiciones de vulnerabilidad y de amenaza, toda vez que se está produciendo un cambio de uso del suelo, que CASTRO $e t$ al., (2009; 2010), concluyó como efectos de carácter negativo. Por otro lado, las formas heredadas del paisaje geológico y morfológico, constituyen a su vez una condición de base propicia para la generación de las amenazas debidas a la pluviometría concentrada de los eventos El Niño. La vulnerabilidad física del territorio constituye una respuesta morfodinámica al impacto generado en el paisaje, sea el natural, como sobre todo, el antropizado (RODOLFI \& ZANCHI 2002). El propósito de este estudio es establecer las 
condiciones de amenaza natural en el valle del río Choapa, centrándose en las localidades de Salamanca y Panguesillo, estableciendo las relaciones con la geomorfología y la intervención del sistema natural.

\section{MATERIALES Y MÉTODOS}

\section{Área de estudio}

El área estudiada corresponde al valle del río Choapa, junto a la ciudad de Salamanca, paisaje inserto en el dominio de la Cordillera de Los Andes (Fig. 1).

\section{Pasos metodológicos}

La definición de unidades geomorfológicas del valle estudiado, fue analizada desde el punto de vista integral, considerando las características del sistema geológico y geomorfológico heredado y actual, como la relación con las actividades que se están llevando a cabo en determinadas áreas y formas. Para lograr un resultado más cuantitativo, transcendiendo a las formas propiamente tales, se recurrió a la modelización de parámetros topográficos a través de herramientas SIG. El esquema metodológico utilizado se presenta en la figura 2.



Fig. 1. Área de estudio. Valle del río Choapa, Región de Coquimbo. Ciudad de Salamanca y localidad de Panguesillo 


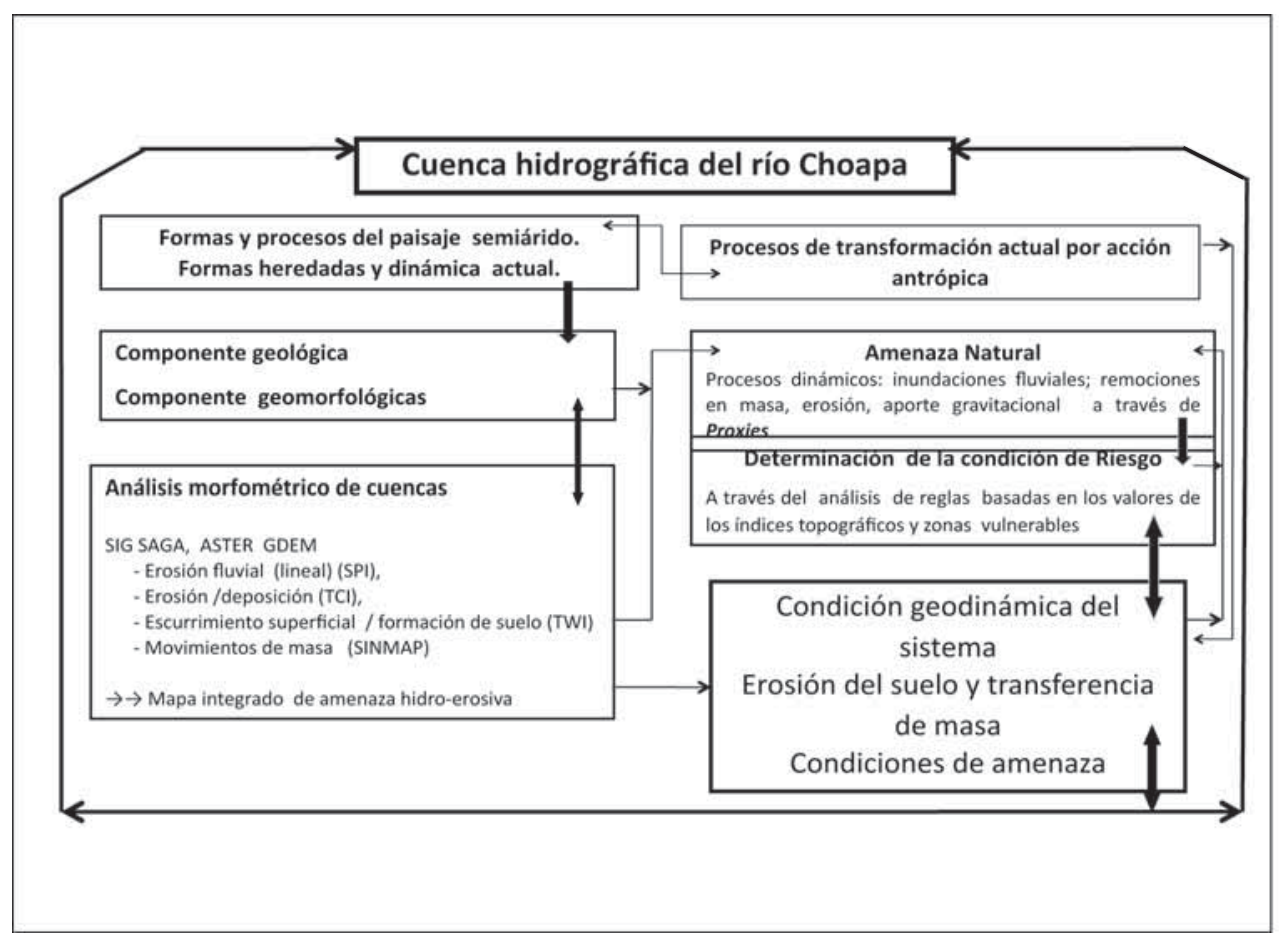

Fig. 2. Esquema metodológico.

Desde el punto de vista de las formas heredadas, se trabajó principalmente con la información de la carta geológica de RIVANO \& SEPÚLVEDA (1991), que aportó la definición de las grandes unidades litológicas y sus edades. A partir de esta información y el trabajo de fotointerpretación y validación en terreno, se generaron las siguientes clases de laderas, depósitos y formas de erosión:

- Laderas en rocas graníticas indiferenciadas.

- Laderas en monoclinales en rocas sedimentarias continentales, y marinas.

- Depósitos aluviales y fluviales: Antiguos (Mioceno); cuaternarios y del Holoceno.

\section{- $\quad$ Glacis (Pediments).}

Estas clases fueron la base para la definición de las unidades geomorfológicas de laderas y depósitos, sean éstos de base de vertientes o las terrazas aluviales. Se elaboró una car- tografía de unidades morfolitológicas para cuenca del río Choapa y de las áreas estudiadas. La base metodológica de la cartografía se sustenta en la clasificación de sistemas de vertientes (ARAYA-VERGARA 1985), y las aplicaciones a diferentes escalas elaboradas por SOTO et al., (2006; 2007) y CASTRO et al., (2009). Las formas y procesos identificados a escala de detalle se realizó de acuerdo a los criterios de ARAYA-VERGARA (1980) y RAPETI et al., (2005). La validación de cartografía geomorfológica se realizó a través de las actividades de terreno.

Complementariamente se trabajó con procesamiento de información digital a través de herramientas SIG. Se realizó un análisis morfométrico aplicando la metodología de MÄRKER et al., (2001, 2008, 2010), y VOGEL \& MÄRKER (2010). Se trabajó en base al análisis de un GDEM ASTER de 30 metros de resolución, corregido hidrológicamente según el algoritmo de PLANCHÓN \& DARBOUX (2001). El DEM preprocesado se utilizó para un análisis del terreno a través del SIG SAGA. 
La evaluación de los procesos de remoción en masa y de erosión hídrica, se elaboró en base a cuatro índices topográficos. Para los fines de la modelización se consideró la presencia de un substrato geológico homogéneo, cualquiera. Los índices se derivaron en consecuencia directamente de la topografía. Para el análisis de los procesos de degradación provocados por la acción del agua y sus efectos en el terreno, las características de escurrimiento son de gran importancia, de la misma manera que la pendiente, que influye en la velocidad de movimiento, mientras que la curvatura, condiciona la convergencia o divergencia de la escorrentía, así como la aceleración de ésta. La pendiente se obtuvo con el algoritmo de ZEVENBERG \& THORNE (1987), siendo un método adecuado para superficies relativamente suaves. Para determinar la dirección y la acumulación de flujo, se aplicó un algoritmo de flujo múltiple (TARBOTON 1997). Los índices combinados permitieron describir las características complejas de la escorrentía (WILSON \& GALLARDO 2000, en MÄKER 2010) y en consecuencia, se ocuparon como indicadores relativos de amenaza natural. El índice Stream Power Index (SPI) se obtuvo como producto de la zona de captación específica y la gradiente. Este índice describe los efectos de los procesos de erosión fluvial lineal, tales como cárcavas, talwegs y erosión lateral de lechos. El índice Topographic Wetness Index (TWI) se calculó como el logaritmo de la proporción específica entre el área de captación y la gradiente. Este índice proporcionó información sobre la acumulación de agua y la saturación del suelo y el substrato, siendo en consecuencia un importante indicador de los procesos de escorrentía superficial e inundaciones. El índice de capacidad de transporte (TCI) según MOORE\& BURCH (1986, en MÄKER 2010), es una aplicación específica en 3D del factor de longitud de la pendiente de la USLE; caracteriza las zonas afectadas por la erosión laminar, transporte de sedimentos y procesos de depositación. El índice de estabilidad (SI) es una implementación específica del índice TWI consi- derando el área de influencia específica y la pendiente (PACK et al., 2001). Este índice caracteriza los peligros de movimientos en masa en el área. La información utilizada en el cálculo de los cuatro índices principales y sus metodologías específicas asociadas se expresan en la tabla 1.

Se realizó la evaluación espacial de los índices señalados. Los valores críticos se obtuvieron mediante la comparación de la distribución espacial del índice único, fotografías aéreas y trabajo de validación en terreno. El trabajo de campo permitió identificar las formas de erosión en las laderas, las coberturas de exhumación de éstas (en vertientes graníticas), evidencias de remoción en masa y marcas de inundación.

Así, para cada uno de los cuatro índices (TWI, SPI, TCI, SI) se derivaron los umbrales respectivos basados en la observación de campo. Los procesos de erosión lineal se identificaron con un SPI mayor a 2500. Las áreas saturadas propensas a la escorrentía superficial e inundaciones, con valores TWI sobre 12,5. Los valores de TCI inferiores a 1 indican aéreas de depositación, mientras que los valores de más de 80 corresponden a erosión. Como el SI muestra valores altos para las partes cuspidales de la zona andina, se consideró adecuado no aplicarlo para la estimación de la amenaza integrada. Los índices individuales relacionados al agua fueron combinados en un mapa final que integró la amenaza natural en dos clases, alta y baja, según el siguiente algoritmo:

donde (SPI $>2500$ o TCI $>80$ o TCI $<1$ o TWI $>$ 12.5), entonces = Alta Amenaza

donde $\mathrm{SPI}<2500$ o $\mathrm{TCI}<80$ o TCI $>1$ o TWI $<12.5$, entonces $=$ Baja Amenaza

Conjuntamente, se representó cartográficamente el trazado urbano actual de las zonas pobladas, en base a fotos aéreas y planimetría urbana. Esta información fue superpuesta a la base de datos SIG, estimando así el alcance 
TABLA I. VARIABLES EMPLEAdAS EN EL MODELAMIENTO DE LA AMENAZA NATURAL, SEGÚN MÄRKER et al. (20I0).

\begin{tabular}{|c|c|}
\hline Variable & Método/Referencia \\
\hline DEM & ASTER GDEM 30m resolution \\
\hline Altitud relativa de red de drenaje & SAGA terrain analysis module (OLAYA \&CONRAD 2008) \\
\hline Elevación & SAGA terrain analysis module (ZEVENBERG \& THORN 1987) \\
\hline Área de la cuenca & SAGA terrain analysis module (OLAYA \& CONRAD 2008) \\
\hline Red de drenaje & SAGA terrain analysis module (OLAYA \& CONRAD 2008) \\
\hline Nivel de base de la red de drenaje & SAGA terrain analysis module (OLAYA \& CONRAD 2008) \\
\hline Convergence index & SAGA terrain analysis module (KOETHE \& LEHMEIER 1996) \\
\hline Curvatura & SAGA terrain analysis module (ZEVENBERG \& THORN 1987) \\
\hline Clasificación de la curvatura & SAGA terrain analysis module (DIKAU 1988) \\
\hline Plano de curvatura & SAGA terrain analysis module (ZEVENBERG \&THORN 1987) \\
\hline Perfil de curvatura & SAGA terrain analysis module (ZEVENBERG \& THORN 1987) \\
\hline Factor LS, TCl & SAGA terrain analysis module (OLAYA \& CONRAD 2008) \\
\hline Laderas & SAGA terrain analysis module (ZEVENBERG \& THORN 1987) \\
\hline Stream power index, SPI & SAGA terrain analysis module (OLAYA \& CONRAD 2008) \\
\hline Subcuenca & SAGA terrain analysis module (OLAYA \& CONRAD 2008) \\
\hline Wetness index, TWI & SAGA terrain analysis module (OLAYA \& CONRAD 2008) \\
\hline Stability Index, SI & ARCGIS 9.2. SINMAP (PACK et al., 2001) \\
\hline
\end{tabular}

territorial de los procesos dinámicos y cómo inciden en las áreas pobladas, pudiendo en consecuencia, identificar las áreas de riesgo.

\section{RESULTADOS}

\section{Paisaje geomorfológico}

El paisaje geomorfológico del valle del río Choapa está constituido por los siguientes grupos de formas: sistemas de laderas en rocas graníticas, formas depositacionales correlativas (conos aluviales), formas de carácter erosional (glacis/pediments), superficies o terrazas fluviales elevadas (Mioceno) y las formas fluviales cuaternarias propiamente tales.

Destacan algunos sistemas de laderas graníticas con y sin presencia de rocas estratifi- cadas (sedimentarias marinas) en las partes cuspidales, situación que desde el punto de vista de los procesos de denudación de las vertientes no da cuenta de una diferenciación entre ambos tipos, en la medida que los depósitos correlativos asociados presentan altura de conos y taludes muy similares (Fig. 3).

Sin embargo, los sistemas de laderas modeladas en rocas graníticas se caracterizan por presentar una densa y continua cobertura detrítica inconsolidada, correspondiente a lo que ARAYA-VERGARA (1980 y 2000) definió como caos de rocas y campos de bloques, respectivamente, y que son el producto de la erosión intensa del granito y la exhumación de la ladera, en bloques esferiodales. Desde la perspectiva de la dinámica actual del paisaje semiárido, este tipo de laderas constituye una condición de amena- 

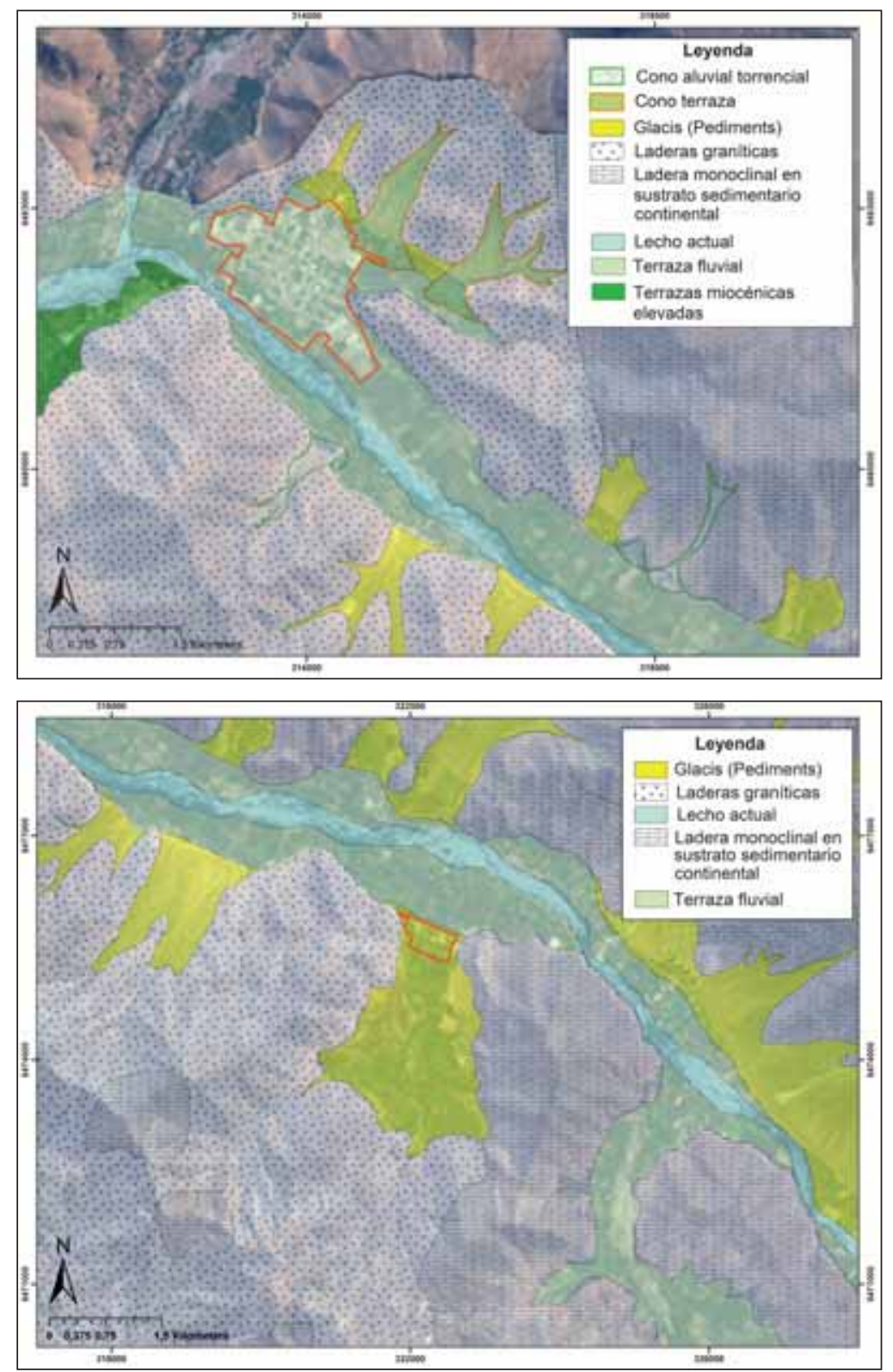

Fig. 3. Carta geomorfológica de Salamanca y Panguesillo. Valle del rio Choapa. Región de Coquimbo.

za a eventos de transporte de masa, flujos de detritos y erosión.

Las formas de conos aluviales están muy bien desarrolladas y son de un marcado carácter torrencial, en función de su fuerte convexidad y el calibre del material en curso. Tanto los conos individuales como aquéllos coalescentes, presentan talwegs muy incididos con material heterométrico disponible en su lecho. Los sistemas de conos asociados a laderas en formas de anfiteatro, presentan una mayor secuencia evolutiva y textural. Tal es el caso del sector de Panguesillo, en que fue posible encontrar paleo suelos en las laderas, gracias a los trabajos de movimiento de tierra realizados para la preparación de plantaciones frutales. 
Un rasgo geomorfológico importante de destacar es la presencia de formas de glacis, con marcadas incisiones y con un funcionamiento dinámico actual ocasional. Estas formas son muy indicativas de las condiciones del paisaje heredado en la cuenca, las que no están en morfogénesis pero si en actividad dinámica. En estas formas, es posible identificar tors y mares de rocas, productos de la intensa erosión. Es importante señalar que si bien el área estudiada corresponde a dos localidades específicas, a lo largo del valle del río Choapa, las superficies de erosión, pediments o glacis y los depositos aluviales, sistemas de conos, son ampliamente recurrentes.

La zona de contacto entre laderas/depósitos de valle está ampliamente representada en la cuenca por superficies o terrazas elevadas de edad miocena, que presentan perfiles de importantes pendientes y superficie muy planiforme. Estas terrazas, de gravas antiguas son muy erosionables y están siendo incorporadas a la agricultura intensiva, realizando en ellas prácticas de nivelación topográfica y trazado de surcos y camellones (Fig. 4).

El fondo del valle presenta sus diferentes niveles de terrazas fluviales del Cuaternario. En términos de los proceso actuales que operan en las diferentes formas ya descritas, lo primero es señalar que se trata de formas heredadas con dinámica actual. Aparte de los procesos gravitacionales de las laderas propios de los ambientes semiáridos, los eventos pluviométricos intensos y concentrados, reactivan todas las formas del paisaje, aportando masa hacia el fondo de valle, las áreas cultivadas y los centros poblados, diferencialmente, en función de tipo de forma involucrada. Evidencias de tales procesos son las marcas de erosión lineal, flujos de detritos y canales torrenciales en laderas, conos y terrazas antropizadas.

En este mismo contexto, las formas debidas a los procesos de depositación cuaternaria, conos aluviales y formas de terrazas fluviales, coexisten con aquéllas debidas a la erosión, tales como las formas de glacis (pedi-

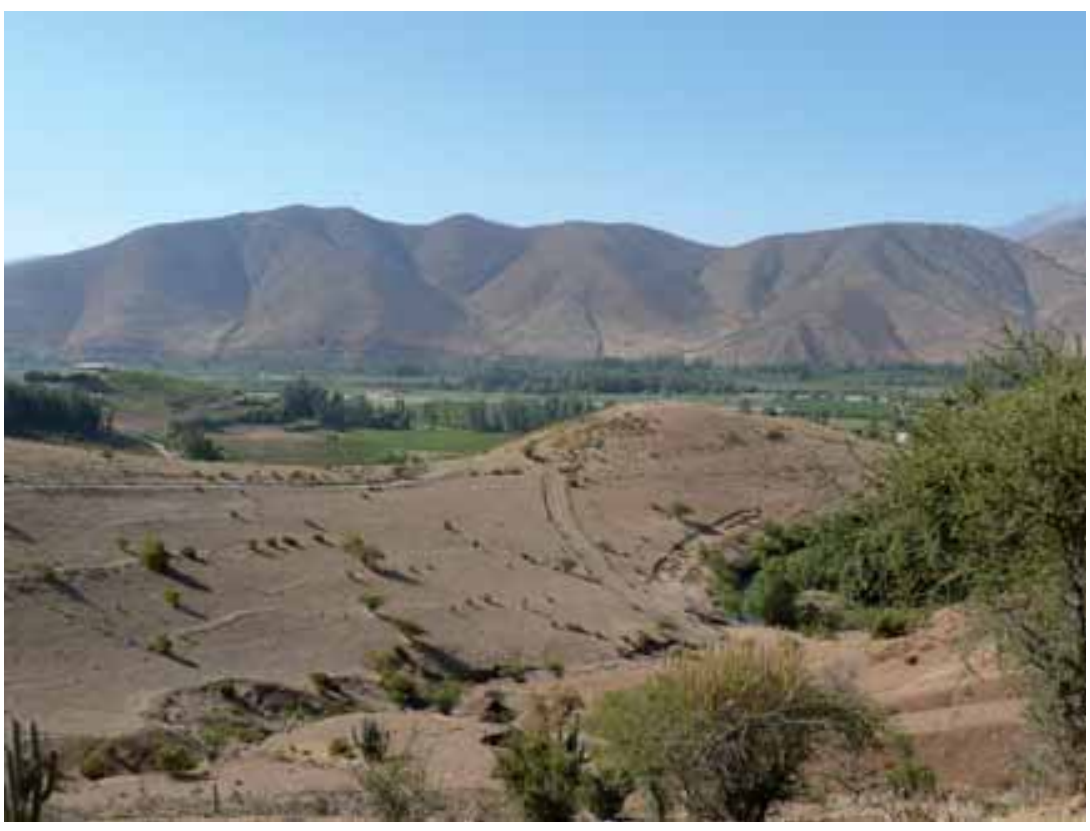

Fig. 4. Terrazas antiguas elevadas (miocénicas). Primer plano, evidencias de erosión y al fondo, producción de frutales. Enero 2009. 
ments). Los fondos de valle y las formas de relleno fluvial son considerablemente diferentes en cuanto a las condiciones de suelos, presentando buenas capacidades de uso.

\section{Condición de amenaza}

La aplicación de los parámetros morfométricos extraídos a partir de la topografía, permitió modelar determinadas condiciones de amenaza, para el entorno de Salamanca y el poblado de Panguesillo. Las condiciones de amenaza a procesos de remoción en masa, expresado a través del stability index, da cuenta que en Salamananca, debido al sistema de laderas de fuerte pendiente y a la presencia de formas de pediment, existen condiciones morfológicas que conforman un paisaje más propenso a las amenazas asociadas a esta clase de fenómenos (Fig. 5). El modelo generado, expresa que las partes altas son muy inestables, asociado a la fuerte pendiente de los estratos rocosos de las partes cuspidales de las laderas. No obstante, estas áreas se constituyen en exportadores de masa que se difunden y disipan en las partes medias de las laderas; los talwegs constituyen vías de difusión morfodinámicamente relevantes, sobretodo porque presentan condiciones de incisión, como rasgo de forma heredada. No obstante lo anterior, el centro poblado se localiza en una unidad aluvial estable, que podría ser receptora de los impactos de las remociones
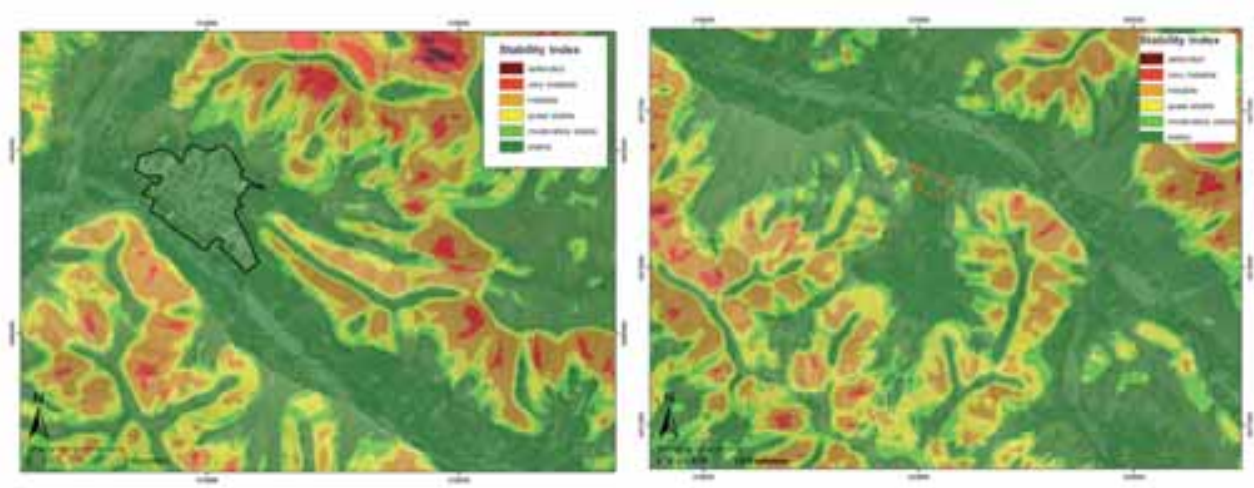

Fig. 5. Modelo de amenaza por remoción en masa. 
sido validadas a través de la geomorfología de campo, que expresan la presencia de sistemas depositacionales de carácter aluvial; sin embargo sobresale la condición de erosión del talweg, que ratifica lo descrito en el modelo anterior. Más aún, la figura 9, expone las condiciones de saturación del suelo y del escurrimiento, la alta amenaza asociada a flujos y escurrimiento superficial a que está sometida la ciudad de Salamanca y también el poblado de Panguesillo. En este último caso, este indicador es el que mejor responde a las condiciones de amenaza esperadas para este sector, en función a las actividades antrópicas que se están desarrollando en ese sector del valle del Choapa.

\section{Determinación de la amenaza natural in- tegrada}

La modelización integrada de los indicadores empleados fue especialmente desarrollada para las condiciones del medio ambiente semiárido de la cuenca, tal como descrito en la metodología. La expresión grafica de este modelo se aprecia en la figura 9, que representa las condiciones de amenaza natural integrada con los parámetros morfométricos y topográficos, para un nivel alto y bajo. Los resultados obtenidos son coherentes con los modelos individuales ya descritos, pero destaca su correlación con las condiciones de geología y geomorfología levantada para el área de estudio. Otro aspecto relevante de validación está asociado a los procesos dinámicos actuales, tales como erosión, evidencias de flujos de detritos, talwegs torrenciales, laderas de recubrimiento detrítico, que no alcanzan a ser representadas por la resolución
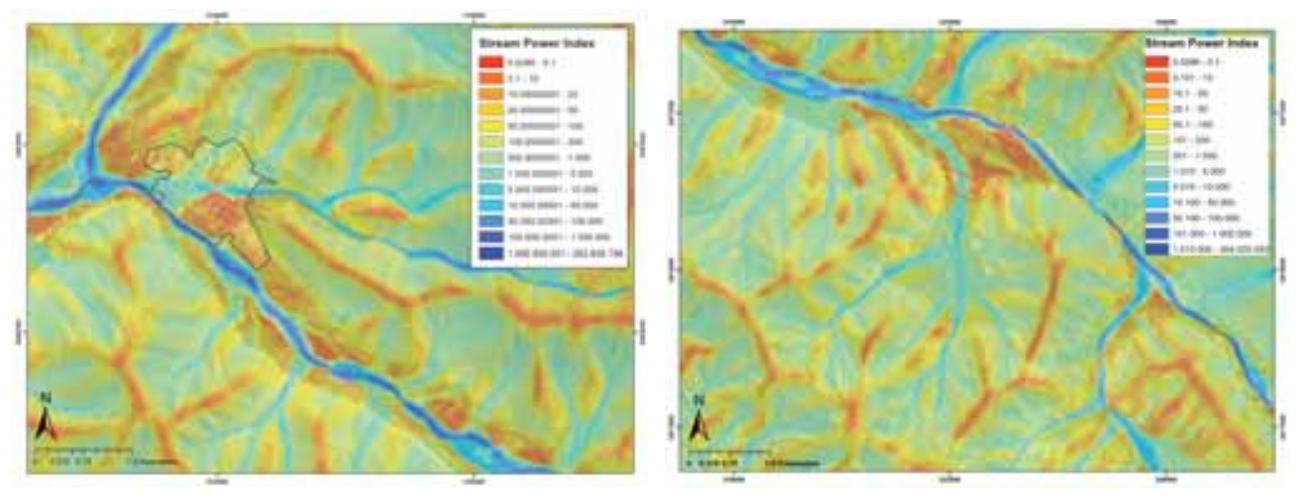

Fig. 6. Modelo de amenaza por erosión fluvial
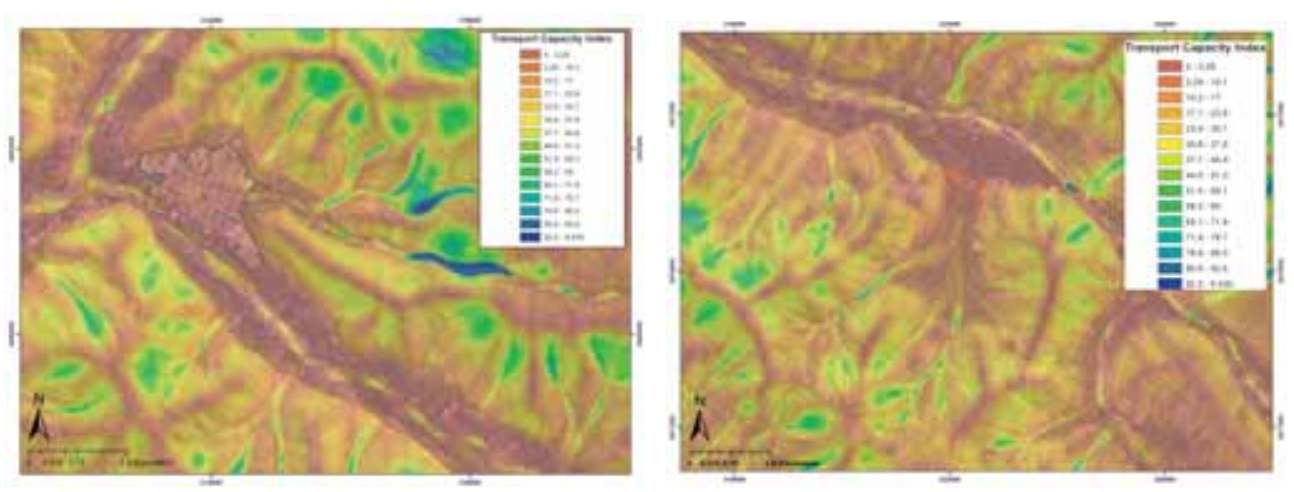

Fig. 7. Modelo de la relación entre erosión y depositación. 

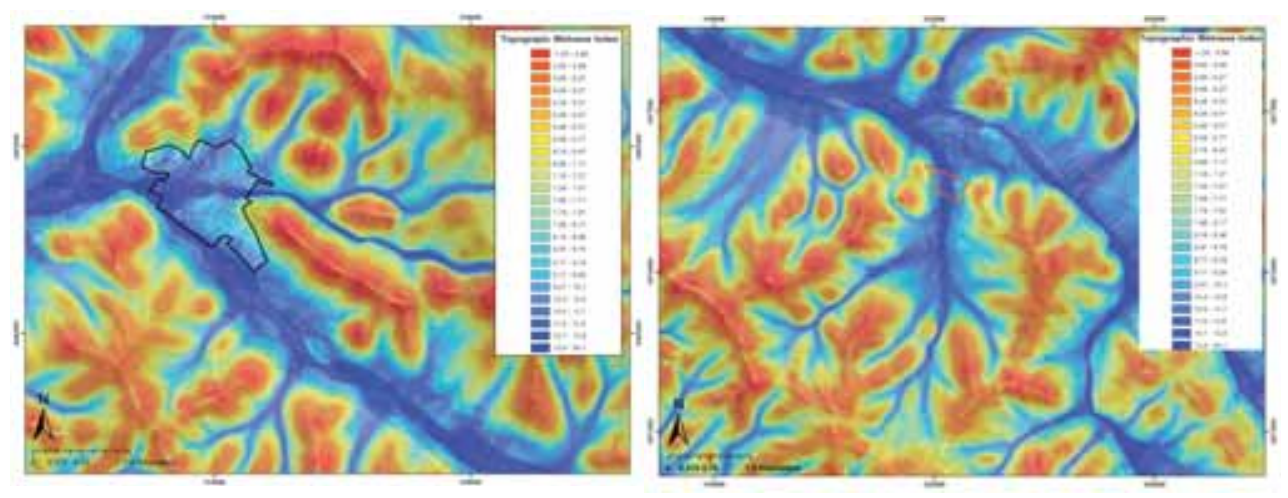

Fig. 8. Modelo de saturación del suelo y escurrimiento.
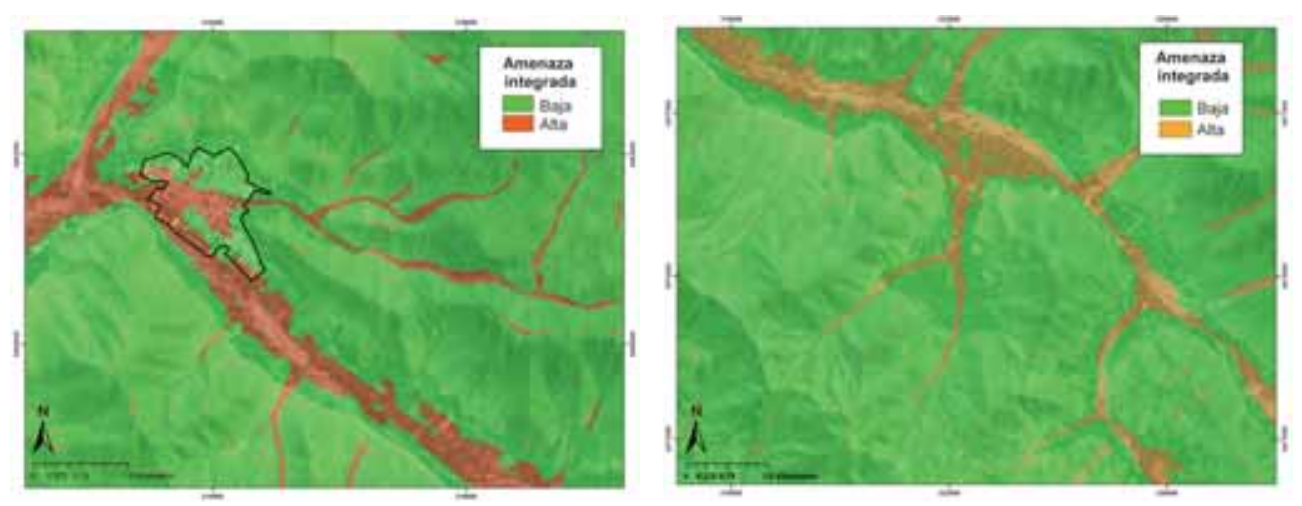

Fig. 9. Modelo de amenaza natural integrada.

del modelo pero que han sido identificadas y mapeadas en terreno.

En virtud de lo anterior, ambas localidades presentan evidentes condiciones de amenaza natural alta, las que se explican por la posición morfológica y topográfica en que se asientan y consecuentemente son vulnerables a procesos vinculados a la acción fluvial, como a la acción de los talwegs y las condiciones de emisarios de flujos (remoción en masa). La situación de amenaza alta de Salamanca se superpone con el área urbana, estableciéndose así el área de población vulnerable y expuesta a una condición de riesgo que habría que evaluar posteriormente.

En Panguesillo la situación de amenaza es similar, pero acrecentada por la condición de intervención de la cuenca, la cual ha sido muy intensa, y está en desarrollo. La figura 10 muestra una visión panorámica del sector, correspondiente a otoño de 2008 , en que se evidencia la fuerte intervención de las laderas y los sistemas de conos aluviales. En la actualidad las prácticas de movimientos de tierra, apertura de caminos, nivelaciones topográficas, desarrollo de surcos y camellones alcanzan hasta las cimas de las laderas. Se asume entonces que la condición de amenaza alta, sería aún más intensa en términos de masa en movimiento ante eventos pluviométricos concentrados. Desde el punto de la vulnerabilidad y el riesgo, este poblado de carácter rural, estaría también expuesto a condiciones de remociones en masa asociadas a flujos. 


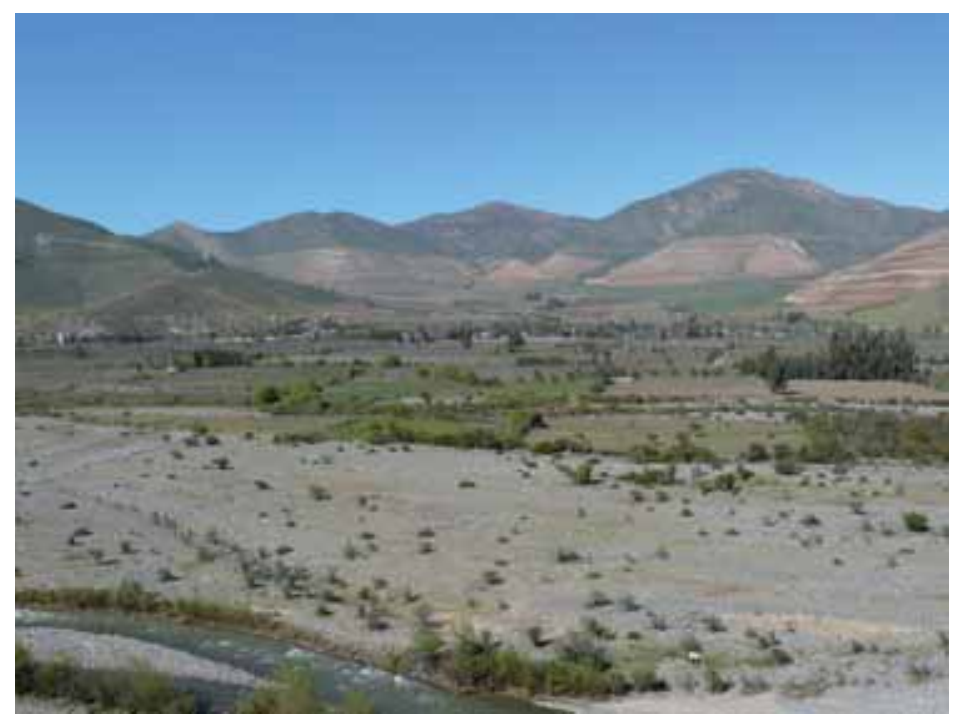

Fig. 10. Vista panorámica de Panguesillo. Otoño 2008. Primer plano, río Choapa.

\section{DISCUSIÓN}

Las características morfológicas del valle del río Choapa en general y de las áreas seleccionadas en especial, en un medio semiárido, dan cuenta de la acción del agua en el modelado de las formas. En este sentido las terrazas del Mioceno (RIVANO \& SEPÚLVEDA 1991), constituyen formas muy relevantes en el valle, tanto desde el punto de vista de la evolución de los procesos geodinámicos, heredados y actuales, como también en términos de las nuevas condiciones dinámicas de estas formas debido a los procesos de intervención física para usos agrícolas intensivos. Esta dinámica ya ha sido descrita para similares unidades en el valle del Copiapó, estableciéndose un nuevo escenario dinámico asociado al aumento de la vulnerabilidad física de estas formas cuando ocurren los eventos El Niño (CASTRO et al., 2009).

De los casos citados se desprende la situación de un domino actual árido y semiárido, pero fuertemente modelado por el agua, durante condiciones climáticas relictas, pero también durante las actuales. Esta aseveración puede ser discutida a la luz de los antecedentes aportados por STRUDLEY et al., (2006) quienes dan cuenta de la intensa meteorización de los substratos graníticos, como en piedmonts y pediments y de la condición de transporte de sedimentos generada. A su vez ANDREW et al., (2008), comprueban la importancia de eventos pluviométricos concentrados, como agentes desencadenantes del transporte de masa en conos aluviales y glacis. Dado que tales hechos morfodinámicos son perfectamente asimilables a los del valle del Choapa, se puede hacer la comparación y deducir una tendencia dinámica correlativa. En este mismo contexto, los cambios observados en la desembocadura del Choapa por ARRIAGADA (2009), donde el río meandrante presentó una clara anastomosis en el período de 40 años, se explica por las condiciones particular ya señaladas, que potencian el transporte de masa desde de la cuenca.

Además, los sistemas de laderas, que se caracterizan por un predominio granítico y de estratos calcáreos culminantes, que presentan un aspecto superficial de cobertura detrítica, serán la resultante de procesos de erosión intensa ya descritos por ARAYAVERGARA (1980, 2000), tanto para una 
sección del valle del Choapa y Chile central, respectivamente, es decir, formas modeladas por la erosión, generando la exhumación de rocas y la alteración de las mismas. Estas características pueden ser interpretadas como una condición propicia de disponibilidad de masa en las laderas para ser removida.

Desde el punto de vista de la dinámica natural se está en presencia de un paisaje activo, fundamentalmente durante eventos pluviométricos intensos y esporádicos. Se evidencia la acción de flujos sobrecargados de masa escurriendo transversalmente al valle, de la misma manera que el aumento violento del caudal del río Choapa. A este escenario natural se deben sumar los cambios de cobertura vegetal, los movimientos de tierra en conos, laderas y terrazas altas, todo lo cual contribuye con lavado de material, erosión y sobrecarga de masa detrítica a los talwgs. Las áreas de influencia de tales procesos coinciden con la localización de centros poblados como Salamanca y Panguesillo.
Los resultados proporcionados a través de los indicadores topográficos empleados, son coherentes con las condiciones dinámicas caracterizadas para la cuenca, los que pueden ser interpretados como condiciones de amenaza natural. Las modelaciones de parámetros topográficos son coherentes con los resultados derivados de la geomorfología local y con las experiencias de MÄRKER et al., (2010) y VOGEL \& MÄRKER (2010). Sin embargo, el análisis se basa sólo en el modelo de terreno, en que todas las variables se mantienen homogéneas (suelos, geología, vegetación). En consecuencia, el método presentado sólo permite una primera aproximación de la distribución espacial de las zonas de peligro o amenaza. La tabla 2 sintetiza la relación entre los indicadores, las condiciones geodinámica y la amenaza consecuente, y se derivan situaciones de amenaza natural y consecuentemente riesgo asociado, en la medida que el área de influencia de los procesos naturales asociados a la amenaza, afecta a los perímetros urbanos de ambas entidades pobladas.

TABLA N ${ }^{\circ}$ 2. RELACIÓN ENTRE PARÁMETROS MORFOMÉTricos, CON LA CONDICIÓN GEODINÁMICA Y LA AMENAZA ASOCIADA

\begin{tabular}{|l|l|}
\hline \multicolumn{1}{|c|}{$\begin{array}{c}\text { Índices } \\
\text { morfométricos }\end{array}$} & \multicolumn{1}{c|}{ Condición geodinámica / Amenaza } \\
\hline $\begin{array}{l}\text { Stability Index, SI } \\
\text { Índice de Estabi- } \\
\text { lidad }\end{array}$ & $\begin{array}{l}\text { Las condiciones favorables para remociones en masa son identificadas en las } \\
\text { partes altas del bloque andino. }\end{array}$ \\
\hline $\begin{array}{l}\text { Stream Power } \\
\text { Index, SPI } \\
\text { Índice de Energía } \\
\text { del Flujo }\end{array}$ & $\begin{array}{l}\text { A excepción de la erosión propia del río Choapa, destaca tanto en el caso de } \\
\text { Salamanaca como Panguesillo la presencia de talwegs que drenan las áreas } \\
\text { pobladas y en consecuencia se deriva una condición de amenaza alta asociada } \\
\text { a eventos pluviométricos torrenciales (El Niño), pues en períodos normales el } \\
\text { caudal de ambos talwegs es irrelevante. }\end{array}$ \\
\hline $\begin{array}{l}\text { Índice de capaci- } \\
\text { dad de transporte } \\
\text { (TCI) }\end{array}$ & $\begin{array}{l}\text { Destaca la condición de los talwegs por su condición de erosión y al mismo } \\
\text { tiempo como emisores, siendo más relevante el caso de Salamanca. En función } \\
\text { a la geomorfología de las subcuencas involucradas, se puede derivar una con- } \\
\text { dición de amenaza baja. }\end{array}$ \\
\hline $\begin{array}{l}\text { Topographic Wet- } \\
\text { ness Index, TWI } \\
\text { Índice de Humedad }\end{array}$ & $\begin{array}{l}\text { Los niveles de disección de los talwegs a portantes a Salamanca y Panguesillo } \\
\text { dan cuenta de una condición altamente favorable para el escurrimiento super- } \\
\text { ficial, que de acuerdo a lo observado por la geomorfología en terreno, estaría } \\
\text { en estrecha relación a eventos torrenciales El Niño. La condición de amenaza } \\
\text { derivada es alta, más aún si se superponen los resultados del Stream Power } \\
\text { Index. }\end{array}$ \\
\hline
\end{tabular}


A este respecto es importante destacar que en la determinación de la condición de amenaza, no se han considerado explícitamente los diferentes grados de intervención en el territorio, el cual es extremo en el caso de Panguesillo y consecuentemente el impacto en las laderas y el suelo es muy alto en términos de la inducción a la erosión, tal como observado por PELLACANI et al., (2006a, 2006b) y CASTRO \& ALIAGA (2010) en cuencas mediterráneas altamente intervenidas por la agricultura.

No obstante lo anterior, a nivel de cuenca, el río Choapa aún no está sujeta a profundos y generalizados cambios de uso del suelo e intensidad del mismo. Los cambios observados están vinculados a actividades extractivas mineras y a una reciente agricultura, muy localizada y altamente intensiva, que no se realiza en los fondo de valle (agricultura tradicional), sino que en áreas de considerable pendiente, de conos aluviales, terrazas del Mioceno y laderas graníticas (ARRIAGADA et al,. 2008). Es importante relacionar estos resultados con aquéllos obtenidos en Salamanca por CASTRO (Comunicación verbal), que señaló que los suelos del área periurbana pueden ser clasificados en el límite de la sostenibilidad, es decir, donde un manejo inadecuado puede llevar al suelo a una condición no sostenible. Si a ello se relacionan los parámetros morfométricos, la geodinámica actual y los potenciales cambios en el paisaje, es posible derivar una condición de exacerbación de la dinámica actual, aumentando la amenaza y consecuentemente la vulnerabilidad y el riesgo a las localidades pobladas analizadas.

Finalmente, hay que destacar que si bien el uso de los indicadores morfométricos entregó resultados bastante coherentes con la realidad geomorfológica de las áreas estudiadas, tal correlación se debe a que tales índices son derivados de la topografía y que una mejor correlación y acercamiento a los procesos deberá realizarse con la inclusión de la variable suelo.

\section{CONCLUSIONES}

Las formas presentes, sistemas de laderas graníticas (principalmente), de conos aluviales torrenciales, terrazas fluviales heredadas del Cuaternario y del Mioceno (terrazas elevadas), y de formas de erosión, como los glacis, poseen un funcionamiento actual intenso, asociado a eventos pluviométricos vinculados a El Niño. Esta situación redunda consecuentemente en la disección de talwegs, erosión lineal en las laderas, intenso arrastre en los glacis, de lo cual se concluye un alto aporte de masa desde tales formas al sistema hidrológico. Ésto se demuestra por el cambio de río eminentemente meandrante a anastomosado, en la sección del exutorio.

De acuerdo a la naturaleza geológica y geomorfológica de las áreas analizadas, se concluye que los cambios de uso del suelo tendiente a un uso intensivo asociado a la preparación de laderas para cultivo de frutales, aportarán una mayor cantidad de masa al sistema.

En término de la modelación SIG empleada, consiste en una metodología simple, que permite una primera aproximación a la evaluación de los riesgos y que pudo ser validada con la geomorfología. La evaluación realizada indica que la principal amenaza y riesgo para los asentamientos poblados analizados está relacionada con los procesos hidrológicos del suelo, como la erosión, los procesos de remoción en masa; saturación y escurrimiento superficial.

Consecuentemente como las amenazas son altas y existe población vulnerable se puede concluir también una condición de riesgo indeterminado. En este mismo contexto, si bien la metodología consideró los rangos extremos, alto y bajo, se plantea la necesidad de establecer en la metodología los niveles intermedios de amenaza. Para ello será necesario también avanzar en observación y medición de terreno junto a los análisis de laboratorio que permita la calibración de las características de suelo con los 
indicadores. Se considera que un análisis en profundidad, considerando la variabilidad espacial de las variables físico ambientales relevantes, permitirá una evaluación detallada de zonas únicas de amenaza o peligro.

\section{REFERENCIAS}

ANDREW, G., EVERITT, B., J.F.M. DUQUE, 2008. Episodic sediment delivery and lanscape conectivity in the Mancos Shale badlands and Fremont River system, Utah, USA. Geomorphology, Vol. 102, 2: 242-251.

ARAYA-VERGARA, J.F, 1980. Evolución de las vertientes y morfogénesis fluvial en el dominio subtropical seco según la carta geomorfológica detallada (Ej: Chile semi árido). Informaciones Geográficas. 27: 3-44

ARAYA-VERGARA, J.F, 1985. Análisis de la carta geomorfológica de la cuenca del Mapocho. Informaciones Geográficas. 32: 31-44.

ARAYA-VERGARA，J.F，2000. Desarrollo de superficies de aplanamiento disectadas sobre coast range en la Región del Maule, Chile central. Informaciones Geográficas. 34: 67-90

ARRIAGADA, J., SOTO, M.V. \& C.P. CASTRO, 2008. Dinámica del complejo estuarial del Choapa: sistema estuario-playa-duna. Región de Coquimbo. En: Resumen XXIX Congreso Nacional de Geografía. Temuco.

ARRIAGADA, J, 2009. Geomorfología estuarial comparada en la zona semiárida de Chile. Casos de Copiapó y Choapa. Tesis para optar al grado de Magíster en Geografía, mención Recursos Territoriales. Facultad de Arquitectura y Urbanismo, Universidad de Chile, Santiago: 100 .
CASTRO, C.P., SOTO, M.V., FERNANDEZ, R., MAERKER, M. y G. RODOLFI, 2009. Impacto de la geodinámica actual del valle de Nantoco, cuenca del río Copiapó, asociado a la reconversión productiva. Revista de Geografía Norte Grande. 42 : 81-99.

CASTRO, C.P. y C. ALIAGA, 2010. Evaluación de la pérdida de suelo, asociada al proceso de expansión urbana y reconversión productiva. Caso: comunas de Los Andes, Quillota y Concón, Valle del Aconcagua. Revista de Geografía Norte Grande. 45: 41-49.

CARDONA. O.D, 2009. Teoría del Riesgos y Desastres. En: Gestión Integral de Riesgos y Desastres. Curso de Educación Superior. Universidad Internacional de Florida. Inédito.

DEANE, A. y P.R. WAYLEN, 2009. ENSO related fluctuations of raifall and their consequences for some rodent populations in north central Chile. Tiempo y Espacio. 23: 105-120.

DIKAU, R, 1988: Entwurf einer geomorphographisch-analytischen Systematik von Reliefeinheiten. Heidelb. Geogr. Baust. 5: 1-45.

GARREAUX, R., ACEITUNO, P., MUÑOZ, R., ROJAS, M. y A. MONTECINOS, 2008. El clima de Chile está cambiando. Comunicación del Proyecto ACT-19. Variabilidad climática en Chile: evaluación, interpretación y proyecciones. Departamento de GF, Universidad de Chile. Santiago.

KÖTHE, R. y F. LEHMEIER, 1996. SARA - Ein Programmsystem zur Automatischen Reliefanalyse. Z. Angew. Geogr. 4: 11-21.

MÄRKER, M.; MORETTI, S. \& RODOLFI, G. 2001. Assessment of water erosion processes and dynamics in semi - arid regions of Southern Africa (kwazulu / Natal, RSA, and Swaziland) using the Erosion Response Units concept (ERU). Geografia Fisica e Dinamica Cuaternaria, No 24: 71 83. 
MÄRKER, M., PELACANI. S. y B. SCHRÖDER, 2010: A functional entity approach to predict soil erosion processes in a small Plio-Pleistocene Mediterranean catchment in Northern Chianti, Italy. DOI: 10.1016/J. Geomorph. 010.10.022.

MÄRKER, M., CASTRO, C.P., PELACANI, S. y M.V. SOTO, 2008. Assesment of degradation susceptibility in the Chacabuco province of central using a morphometric based response units approach. Geografia Física e Dinamica Quaternaria. 31: 47-53.

OLAYA, V. y O. CONRAD, 2008. Geomorphometry in SAGA. In: Hengl, T., Reuter, H.I. (Eds.), Geomorphometry: Concepts, Software, Applications. Elsevier, Amsterdam: 293-308.

PACK, R.T., TARBOTON, D.G. y C.N. GOODWIN, 2001. Assessing Terrain Stability in a GIS using SINMAP. In: 15th annual GIS conference, GIS 2001, Vancouver, British Columbia, February :19-22.

PELACANI, S., UNGARO, F., LOMBARDI, L y G. RODOLFI, 2006a. Applicazione di techiche geostatistiche e GIS per la caratterizzazione dei suoli e della loro erodabilita. In: RODOLFI, G. Water erosion in mediterranean environment: direct and indirect assessment in test areas and catchments. $1^{\circ} \mathrm{Ed}$. Genova: Ministero dell Universita e Della Ricerca Progetti di Rilevante Interesse Nazionale (PRIN/COFIN 2002). Brigati, Genova.

PELACANI, S., LOMBARDI, L., MAERKER, M y G. RODOLFI, 2006b. Caratterizzazione dei processi geomorfici di versante nel bacino del torrente orme (Toscana Centrale) sulla base delle unita di risposta erosiva - ERU. Ministero dell Universita e Della Ricerca Progetti di Rilevante Interesse Nazionale (PRIN/COFIN 2002). Brigati, Genova.

RIVANO, S. y P. SEPÚlVEDA, 1991. Carta Geológica de Chile, Hola Illapel, Región de Coquimbo. Servicio Nacional de Geología y Minería. № 69.
RAPETI, F., SALVETTI, M. y M. SPAGNARO, 2005. Carta geomorfológica del bacino del T. Roglio (Vald'Era-Tosacana). En : Rodolfi, G., Dessena, M., Vacca, s. Il sistema idrografico Flumendosa-Mulargia : le acque, il suoli e i processi erosivi. Cenova : Ediciones de la Universitá degli Studi di Sassari.

HELMHOLTZ ASSOCIATION, 2007. Risk Habitat Megacity. In: Proyecto Risk Habitat Megacity. ¿Sostenibilidad en Riesgo? A Helmholtz Research Initiative, 2007 2013. Research Plan I. march 2007. Inédito.

RODOLFI, G. y C. ZANCHI, 2002. Cultures viticoles et phénomenes d'instabilité géomorpholique en Italie centrale. Reveue officielle de l'Union Francaises des Geologues. 135: 114.121.

SOTO, M.V., CASTRO, C.P., RODOLFI, G., MAERKER, M., FERNANDEZ, R., PADILLA, R. y V. RUGIERO, 2007. Carta geomorfológica de la sección central y occidental de la Región Metropolitana de Santiago. 39 : 91-99.

\section{SOTO, M.V.; CASTRO. C.P.; MÄRKER,} M.; RODOLFI, G. \& J. ARRIAGADA. 2009. Procesos dinámicos actuales del semiárido chileno asociado a la generación de condiciones de riesgo. Salamanca, Región de Coquimbo. En: Actas XII Congreso Geológico Chileno. Publicación Digital. Santiago.

STRUDLEY, M., MURRAY, A.B. y P.K. HAFF, 2006. Emergence of pediments, tors, and piedmont junctions from a bedrock weathering-regolith thickness feedback. Geology. Vol. 34: 10, 805-808.

VOGEL, S. y M. MÄRKER, 2010. Reconstruction of the pre-Eruption AD 79 Paleo-topography and Paleo-environment of the Sarno River Basin (Italy) using Stratigraphical Core Drillings and Data Mining Technologies. Geomorphology Vol. 115, 1-2: 67-77. 
ZEVENBERGEN L.W. y C.R. THORNE, 1987. Quantitative Analysis of Land Surface Topography. Earth Surf. Proc. Land. 12: 4756.

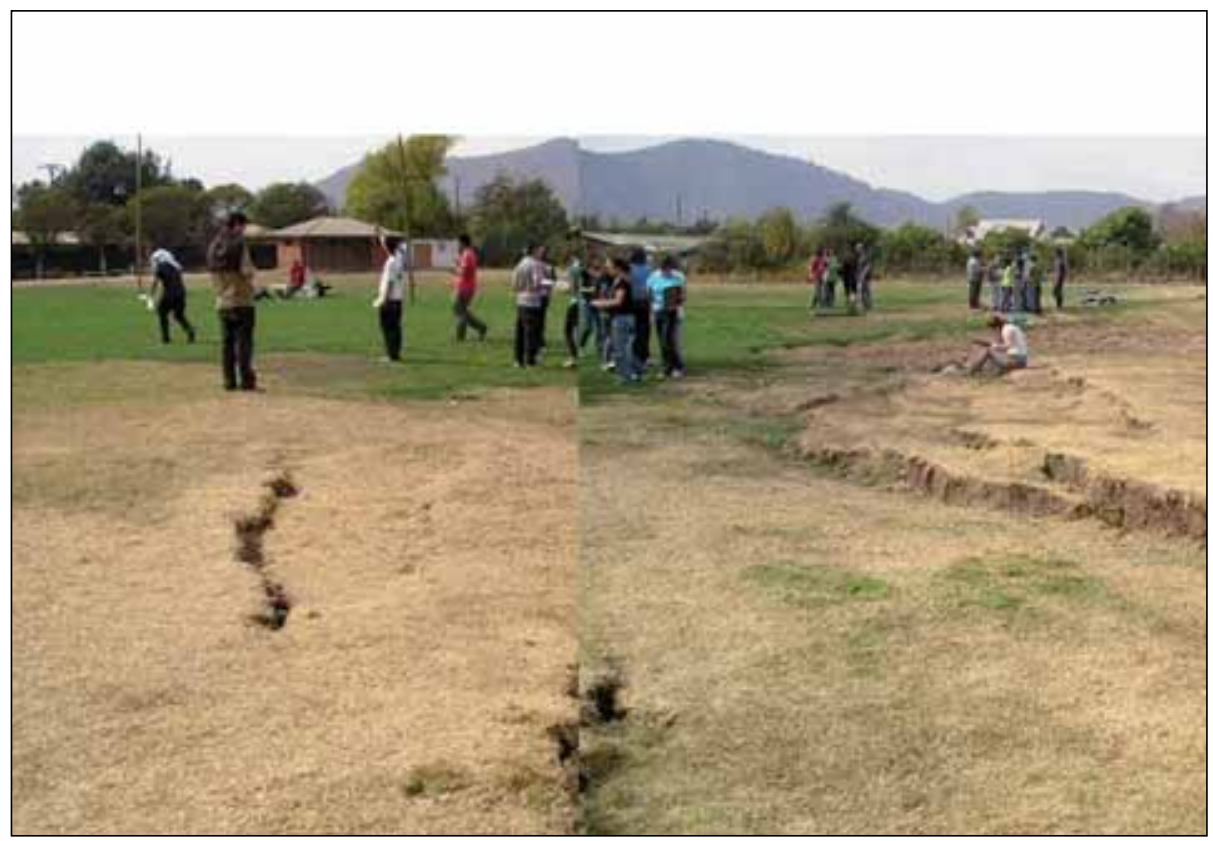

Deformación tectónica co-sísmica de terraza fluvial durante el sismo de 27 de febrero de 2010. Ejemplo: terraza del Maipo en San Antonio de Naltagua, Chile Central (3344’04”S-7101'20”W). Mini rift valley interpretado siguiendo el modelo derivado del experimento de CLOSS (1931). Observaciones de campo indicaron a este autor que la dirección de un esfuerzo impuesto influye en la orientación de un sistema de fracturas, si una masa sedimentaria es abombada. Este proceso produce graben y, consecuentemente, un rift valley por extensión, acompañado también de horsts internos. Un ejemplo gigantesco de este fenómeno es el Rift Valley o Gran Grieta Africana.

Co-seismic tectonic deformation of fluvial terrace during the seism of february 27, 2010. Example: Maipo terrace in San Antonio de Naltagua, Central Chile (33॰44'04”S-7101'20”W). Mini rift valley interpreted following the model derived from an experiment by CLOSS (1931). Field observations indicated to this author that the direction of an imposed stress influences on the orientation of a fracture system if a sedimentary mass suffers a convex deformation. This process produces graben and, consequently, a rift valley by extension, accompanied also by internal horsts. A gigantic example of this phenomenon is the Rift Valley or Great African Crack. Referencia: CLOSS, H.,1931. Zur experimentellen Tectonic II. Brüche und Falten. Naturwissenschaften, 19: 242-247.

Texto y foto: José F. Araya Vergara (c) 2010 Invest. Geogr. Chile. Todos los derechos reservados. 